\title{
The state of commercial augmentative biological control: plenty of natural enemies, but a frustrating lack of uptake
}

\author{
Joop C. van Lenteren
}

Received: 20 March 2011 / Accepted: 7 July 2011 / Published online: 28 July 2011

(C) The Author(s) 2011. This article is published with open access at Springerlink.com

\begin{abstract}
Augmentative biological control concerns the periodical release of natural enemies. In commercial augmentative biological control, natural enemies are mass-reared in biofactories for release in large numbers to obtain an immediate control of pests. The history of commercial mass production of natural enemies spans a period of roughly 120 years. It has been a successful, environmentally and economically sound alternative for chemical pest control in crops like fruit orchards, maize, cotton, sugar cane, soybean, vineyards and greenhouses. Currently, augmentative biological control is in a critical phase, even though during the past decades it has moved from a cottage industry to professional production. Many efficient species of natural enemies have been discovered and 230 are commercially available today. The industry developed quality control guidelines, mass production, shipment and release methods as well as adequate guidance for farmers. However, augmentative biological control is applied on a frustratingly small acreage. Trends in research and application are reviewed, causes explaining the limited uptake are discussed and ways to increase
\end{abstract}

Handling Editor: Stefano Colazza

J. C. van Lenteren $(\square)$

Laboratory of Entomology, Wageningen University, P.O. Box 8031, $6700 \mathrm{EH}$ Wageningen, The Netherlands e-mail: Joop.vanLenteren@wur.nl application of augmentative biological control are explored.

Keywords Exotic natural enemies · Indigenous natural enemies - Trends in natural enemy use . Pesticide substitution - Access and benefit sharing in biological control

\section{Introduction}

Biological control is the use of an organism to reduce the population density of another organism. Biological control has been in use for about two millennia, and has become widely used in pest management since the end of the nineteenth century (DeBach 1964; van Lenteren and Godfray 2005). The following types of biological control can be distinguished: natural, conservation, inoculative (=classical) and augmentative biological control. Natural biological control is the reduction of pest organisms by their natural enemies and has been occurring since the evolution of the first terrestrial ecosystems some 500 million years ago. It takes place in all of the world's ecosystems without any human intervention, and, in economic terms, is the greatest contribution of biological control to agriculture (Waage and Greathead 1988). Conservation biological control consists of human actions that protect and stimulate the performance of naturally occurring natural enemies (Gurr and Wratten 2000). In inoculative biological control, natural enemies are collected in an 
exploration area (usually the area of origin of the pest) and then released in new areas where the pest was accidentally introduced. The aim is that the offspring of the released natural enemies build up populations which are large enough for suppression of pest populations during many subsequent years. This type of biological control has been used most frequently against introduced pests, which are presumed to have arrived in a new area without their natural enemies. As it was the first type of biological control practised widely, it is also called "classical" biological control (DeBach 1964). In augmentative biological control natural enemies are mass-reared in biofactories for release in large numbers to obtain an immediate control of pests. The history of commercial mass production and sale of natural enemies spans a period of roughly 120 years. In some areas of agriculture, such as fruit orchards, maize, cotton, sugarcane, soybean, vineyards and greenhouses, it has been an environmentally and economically sound successful alternative to chemical pest control (van Lenteren and Bueno 2003). Natural, conservation and inoculative biological control are generally carried out using public funding, whereas augmentative biological control is often a commercial activity because of the need of mass production and large scale regular releases of natural enemies.

Inoculative biological control is estimated to be used on $10 \%$ of land under cultivation (Bale et al. 2008) and, over the last 120 years, 165 pest species have been brought under long-term control (Cock et al. 2010). Cock et al. (2010) estimated that worldwide 170 species of invertebrate natural enemies are produced and sold globally for periodical release in augmentative biological control of more than 100 pest species on about $0.4 \%$ of land under cultivation. Augmentative biological control is operated by state-funded or commercial biofactories (van Lenteren and Bueno 2003).

Currently, augmentative biological control is in a critical phase, even though during the past decades it has moved from a cottage industry to professional production, which has identified many efficient species of natural enemies, developed quality control protocols, mass production, shipment and release methods, as well as adequate guidance for farmers (van Lenteren 2003; Cock et al. 2010). Large recent successes, such as the virtually complete replacement of pesticides by predatory mites to control thrips in
Spain, show how well biological control can function (Merino-Pachero 2007) and literally saved vegetable production. However, this form of biological control is applied on a frustratingly small acreage, even though biological control has been considered the environmentally safest and most economically profitable form of pest management (e.g. DeBach and Rosen 1991; Cock et al. 2010). Thus, the question emerges "which factors prevent a much larger use of augmentative biological control?"

This paper first provides information about the 230 species of natural enemies which are used presently in augmentative biological control. Next, I explore why this type of biological control is not used more often. Finally, I summarize developments which are expected to lead to increased application of augmentative biological control.

\section{Natural enemies used in augmentative biological control worldwide}

Cock et al.'s (2010) database lists more than 170 species of invertebrate natural enemies that are used in augmentative biological control in Europe. Information about use of augmentative biological control outside Europe was obtained from recent literature and personal contacts. Although it was difficult to get hold of recent data for some areas of the world (e.g. several large Asian countries and Russia), Table 1 probably includes more than $95 \%$ of species used in augmentative releases, and allows a number of important conclusions to be drawn.

In 2010, no less than 230 species of invertebrate natural enemies-originating from ten taxonomic groups-were used in pest management worldwide. The majority of species belongs to the Arthropoda (219 out of 230 species $=95.2 \%$ ) and only 11 species (one belonging to the Mollusca and ten belonging to the Nematoda) are non-arthropods. Within the arthropods, four taxonomic groups provided most natural enemies: first of all the Hymenoptera $(52.2 \%, 120$ species), next the Acari (13.1\%, 30 species), followed by the Coleoptera $(12.2 \%, 28$ species) and Heteroptera (8.3\%, 19 species) (Fig. 1). The large number of hymenopteran species used in augmentative control can be explained as follows: compared to predators, hymenopteran parasitoids are more specific and, therefore, have a much more 
Table 1 Commercial availability of invertebrate natural enemies used worldwide in augmentative biological control, with region of use, year of first use and market value

\begin{tabular}{|c|c|c|c|c|c|}
\hline Natural enemy & Classification & Region where used & Target(s) & $\begin{array}{l}\text { Year of } \\
\text { first use } \\
\text { (estimated) }\end{array}$ & $\begin{array}{l}\text { Market } \\
\text { value }\end{array}$ \\
\hline Adalia bipunctata & Coleoptera & Europe, North America & Aphids & 1998 & $\mathrm{~S}$ \\
\hline Aleochara bilineata & Coleoptera & Europe & Root flies & 1995 & $\mathrm{~S}$ \\
\hline Aeolothrips intermedius & Thysanoptera & Europe & Thrips & 2000 & $\mathrm{~S}$ \\
\hline Aleurodothrips fasciapennis & Thysanoptera & Europe & Diaspidids & 1990 & $\mathrm{~S}$ \\
\hline $\begin{array}{l}\text { Amblyseius andersoni } \\
\quad(=\text { potentillae })\end{array}$ & Acari & Europe, North America, Asia & Mites & 1995 & $\mathrm{~S}$ \\
\hline Amblyseius largoensis & Acari & Europe & Mites & 1995 & $\mathrm{~S}$ \\
\hline Amblyseius limonicus & Acari & Europe & Mites, thrips & 1995 & $\mathrm{~S}$ \\
\hline Amblyseius swirskii & Acari & $\begin{array}{l}\text { Europe, Africa North and } \\
\text { South, North and Latin } \\
\text { America, Asia }\end{array}$ & $\begin{array}{l}\text { Mites, thrips, } \\
\text { whiteflies }\end{array}$ & 2005 & $\mathrm{~L}$ \\
\hline Amblyseius womersleyii & Acari & Asia & Mites & 2005 & $\mathrm{~L}$ \\
\hline Ampulex compressa & Hymenoptera & Europe & Cockroaches & 1990 & $\mathrm{~S}$ \\
\hline Anagrus atomus & Hymenoptera & Europe & Cicadellids & 1990 & $\mathrm{~S}$ \\
\hline Anagyrus dactylopii & Hymenoptera & Europe & Pseudococcids & 1995 & $\mathrm{~S}$ \\
\hline Anagyrus fusciventris & Hymenoptera & Europe & Pseudococcids & 1995 & $\mathrm{~S}$ \\
\hline Anagyrus pseudococci & Hymenoptera & Europe, North America & Pseudococcids & 1995 & $\mathrm{~S}$ \\
\hline Anaphes iole & Hymenoptera & Europe & Heteropterans & 1990 & $\mathrm{~S}$ \\
\hline Anthocoris nemoralis & Heteroptera & Europe, North America & Psyllids & 1990 & $\mathrm{~S}$ \\
\hline Anthocoris nemorum & Heteroptera & Europe & Psyllids, thrips & 1992 & $\mathrm{~S}$ \\
\hline Aphelinus abdominalis & Hymenoptera & $\begin{array}{l}\text { Europe, Africa North, North } \\
\text { America, Asia }\end{array}$ & Aphids & 1992 & M \\
\hline Aphelinus asychis & Hymenoptera & Asia & Aphids & 2005 & $\mathrm{~S}$ \\
\hline Aphelinus mali & Hymenoptera & Europe & Aphids & 1980 & $\mathrm{~S}$ \\
\hline Aphelinus varipes & Hymenoptera & Europe & Aphids & 2000 & $\mathrm{~S}$ \\
\hline Aphidius colemani & Hymenoptera & $\begin{array}{l}\text { Europe, Africa North and } \\
\text { South, North America, Asia, } \\
\text { Aus/NZ }\end{array}$ & Aphids & 1991 & $\mathrm{~L}$ \\
\hline Aphidius ervi & Hymenoptera & $\begin{array}{l}\text { Europe, Africa North, North } \\
\text { and Latin America, Asia }\end{array}$ & Aphids & 1996 & $\mathrm{~L}$ \\
\hline Aphidius gifuensis & Hymenoptera & Asia & Aphids & 2005 & M \\
\hline Aphidius matricariae & Hymenoptera & Europe, North America & Aphids & 1980 & M \\
\hline Aphidius transcaspinus & Hymenoptera & Africa South & Aphids & 2005 & M \\
\hline Aphidius urticae & Hymenoptera & Europe & Aphids & 1990 & $\mathrm{~S}$ \\
\hline Aphidoletes aphidimyza & Diptera & $\begin{array}{l}\text { Europe, Africa North and } \\
\text { South, North America, Asia }\end{array}$ & Aphids & 1989 & $\mathrm{~L}$ \\
\hline Aphytis diaspidis & Hymenoptera & Europe & Diaspidids & 1990 & $\mathrm{~S}$ \\
\hline Aphytis holoxanthus & Hymenoptera & Europe & Diaspidids & 1996 & $\mathrm{~S}$ \\
\hline Aphytis lepidosaphes & Hymenoptera & Europe & Diaspidids & 1985 & $\mathrm{~S}$ \\
\hline Aphytis lingnanensis & Hymenoptera & Europe, Africa South, Aus & Diaspidids & 1906 & $\mathrm{~L}$ \\
\hline Aphytis melinus & Hymenoptera & Europe, North America, Aus & Diaspidids & 1961 & $\mathrm{~L}$ \\
\hline Aphytis spp. Peru & Hymenoptera & Latin America & Diaspidids & 1990 & M \\
\hline Aprostocetus hagenowii & Hymenoptera & Europe & Cockroaches & 1990 & $\mathrm{~S}$ \\
\hline
\end{tabular}


Table 1 continued

\begin{tabular}{|c|c|c|c|c|c|}
\hline Natural enemy & Classification & Region where used & Target(s) & $\begin{array}{l}\text { Year of } \\
\text { first use } \\
\text { (estimated) }\end{array}$ & $\begin{array}{l}\text { Market } \\
\text { value }\end{array}$ \\
\hline Arrhenophagus albitibiae & Hymenoptera & Europe & Diaspidids & 1990 & $\mathrm{~S}$ \\
\hline Blastothrix brittanica & Hymenoptera & Europe & Coccids & 2005 & $\mathrm{~S}$ \\
\hline Bracon hebetor & Hymenoptera & Europe, North America & Lepidopterans & 1980 & $\mathrm{~S}$ \\
\hline Brontocoris tabidus & Heteroptera & Latin America & Lepidopterans & 1990 & $\mathrm{~S}$ \\
\hline Cales noacki & Hymenoptera & Europe & Whiteflies & 1970 & $\mathrm{~S}$ \\
\hline Carcinops pumilio & Coleoptera & North America & Dipterans & 1990 & $\mathrm{~S}$ \\
\hline Cephalonomia stephanoderis & Hymenoptera & Latin America & Coleopterans & 1990 & $\mathrm{~L}$ \\
\hline Chilocorus baileyi & Coleoptera & Europe, Aus & Diaspidids & 1992 & $\mathrm{~S}$ \\
\hline Chilocorus bipustulatus & Coleoptera & Europe & Diaspidids & 1992-2005 & $\mathbf{S}$ \\
\hline Chilocorus circumdatus & Coleoptera & Europe, Aus & Diaspidids & 1902 & $\mathrm{~S}$ \\
\hline Chilocorus nigritus & Coleoptera & Europe, Africa South & Diaspidids & 1985 & $\mathrm{~S}$ \\
\hline $\begin{array}{l}\text { Chrysoperla }(=\text { Chrysopa }) \\
\text { carnea }\end{array}$ & Neuroptera & $\begin{array}{l}\text { Europe, Africa North, North } \\
\text { and Latin America, Asia }\end{array}$ & Aphids & 1970 & M \\
\hline Chryosperla externa & Neuroptera & Latin America & Lepidopterans & 1980 & $\mathrm{~L}$ \\
\hline Chrysoperla spp. Peru & Neuroptera & Latin America & Aphids & 1990 & $\mathrm{~L}$ \\
\hline Chrysoperla rufilabris & Neuroptera & Europe, North America & Aphids & 1970 & $\mathrm{~S}$ \\
\hline Clitostethus arcuatus & Coleoptera & Europe & Whiteflies & 1997 & $\mathrm{~S}$ \\
\hline Coccidencyrtus ochraceipes & Hymenoptera & Europe & Diaspidids & 1995 & $\mathrm{~S}$ \\
\hline Coccidoxenoides perminutus & Hymenoptera & $\begin{array}{l}\text { Europe, Africa North and } \\
\text { South }\end{array}$ & $\begin{array}{l}\text { Diaspidids, } \\
\text { pseudococcids }\end{array}$ & 1995 & $\mathrm{~S}$ \\
\hline Coccinella septempunctata & Coleoptera & Europe & Aphids & 1980 & $\mathrm{~S}$ \\
\hline Coccophagus cowperi & Hymenoptera & Europe & $\begin{array}{l}\text { Coccids, } \\
\text { pseudococcids }\end{array}$ & 1985 & $\mathrm{~S}$ \\
\hline Coccophagus gurneyi & Hymenoptera & Europe & $\begin{array}{l}\text { Diaspidids, } \\
\text { pseudococcids }\end{array}$ & 1985 & $\mathrm{~S}$ \\
\hline Coccophagus lycimnia & Hymenoptera & Europe & Coccids & 1988 & $\mathrm{~S}$ \\
\hline Coccophagus pulvinariae & Hymenoptera & Europe & Coccids & 1990 & $\mathrm{~S}$ \\
\hline Coccophagus rusti & Hymenoptera & Europe & Coccids & 1988 & $\mathrm{~S}$ \\
\hline Coccophagus scutellaris & Hymenoptera & Europe & Coccids & 1986 & $\mathrm{~S}$ \\
\hline Coccophagus spp. Peru & Hymenoptera & Latin America & Coccids & 1990 & M \\
\hline Coenosia attenuata & Diptera & Europe & Dipterans, whiteflies & 1996 & $\mathrm{~S}$ \\
\hline Comperiella bifasciata & Hymenoptera & Europe & Diaspidids & 1985 & $\mathrm{~S}$ \\
\hline Coniopteryx tineiformis & Neuroptera & Europe & $\begin{array}{l}\text { Aphids, mites, } \\
\text { scales }\end{array}$ & 1990-2005 & $\mathbf{S}$ \\
\hline Conwentzia psociformis & Neuroptera & Europe & $\begin{array}{l}\text { Aphids, mites, } \\
\text { scales }\end{array}$ & 1990-2005 & $\mathbf{S}$ \\
\hline Cotesia flavipes & Hymenoptera & Latin America & Lepidopterans & 1974 & $\mathrm{~L}$ \\
\hline Cotesia glomerata & Hymenoptera & Europe & Lepidopterans & 1995 & $\mathrm{~S}$ \\
\hline Cotesia rubecola & Hymenoptera & Europe & Lepidopterans & 2000 & $\mathrm{~S}$ \\
\hline Cryptolaemus montrouzieri & Coleoptera & $\begin{array}{c}\text { Europe, Africa North and } \\
\text { South, North and Latin } \\
\text { America, Asia, Aus/NZ }\end{array}$ & $\begin{array}{l}\text { Coccids, } \\
\text { pseudococcids }\end{array}$ & 1917 & $\mathrm{~L}$ \\
\hline Cybocephalus nipponicus & Coleoptera & North America & Scales & 2000 & $\mathrm{~S}$ \\
\hline
\end{tabular}


Table 1 continued

\begin{tabular}{|c|c|c|c|c|c|}
\hline Natural enemy & Classification & Region where used & Target(s) & $\begin{array}{l}\text { Year of } \\
\text { first use } \\
\text { (estimated) }\end{array}$ & $\begin{array}{l}\text { Market } \\
\text { value }\end{array}$ \\
\hline Dacnusa sibirica & Hymenoptera & $\begin{array}{l}\text { Europe, Africa North, North } \\
\text { and Latin America, Asia }\end{array}$ & Dipterans & 1981 & $\mathrm{~L}$ \\
\hline Dalotia (Atheta) coriaria & Coleoptera & $\begin{array}{l}\text { European, North America, } \\
\text { Asia, Aus }\end{array}$ & Dipterans, thrips & 2000 & $\mathrm{~S}$ \\
\hline Delphastus catalinae & Coleoptera & Europe, North America & Whiteflies & 1985 & $\mathrm{~S}$ \\
\hline Delphastus pusillus & Coleoptera & Europe, North America & Whiteflies & 1993 & M \\
\hline Digliphus begini & Hymenoptera & Latin America & Dipterans & 2000 & M \\
\hline Dicyphus errans & Hymenoptera & Europe & Dipterans & 2000 & $\mathrm{~S}$ \\
\hline Diglyphus isaea & Hymenoptera & $\begin{array}{l}\text { Europe, Africa North and } \\
\text { South, North and Latin } \\
\text { America, Asia }\end{array}$ & Dipterans & 1984 & $\mathrm{~L}$ \\
\hline Dicyphus hesperus & Hymenoptera & Europe & Whiteflies & 2000-2005 & $\mathbf{L}$ \\
\hline Dicyphus hesperus & Hymenoptera & North America & Whiteflies & 1995 & M \\
\hline Diomus spec. & Coleoptera & Europe & Scales & 1990 & $\mathrm{~S}$ \\
\hline Encarsia citrina & Hymenoptera & Europe & Diaspidids & 1984 & $\mathrm{~S}$ \\
\hline Encarsia guadeloupae & Hymenoptera & Europe & Whiteflies & 1990-2000 & $\mathbf{S}$ \\
\hline Encarsia hispida & Hymenoptera & Europe & Whiteflies & 1990-2000 & $\mathbf{S}$ \\
\hline Encarsia formosa & Hymenoptera & $\begin{array}{c}\text { Europe, Africa North and } \\
\text { South, North and Latin } \\
\text { America, Asia, Aus/NZ }\end{array}$ & Whiteflies & 1926 & $\mathrm{~L}$ \\
\hline Encarsia protransvena & Hymenoptera & Europe & Whiteflies & 1990-2005 & $\mathbf{S}$ \\
\hline Encarsia tricolor & Hymenoptera & Europe & Whiteflies & 1985 & $\mathrm{~S}$ \\
\hline Encyrtus infelix & Hymenoptera & Europe & Coccids & 1990 & $\mathrm{~S}$ \\
\hline Encyrtus lecaniorum & Hymenoptera & Europe & Coccids & 1985 & $\mathrm{~S}$ \\
\hline Episyrphus balteatus & Diptera & Europe & Aphids & 1990 & M \\
\hline Eretmocerus corni & Hymenoptera & Latin America & Whiteflies & 2000 & $\mathrm{~S}$ \\
\hline Eretmocerus eremicus & Hymenoptera & Europe & Whiteflies & 1995-2002 & $\mathbf{L}$ \\
\hline Eretmocerus eremicus & Hymenoptera & $\begin{array}{l}\text { Africa North and South, North } \\
\text { and Latin America, Asia }\end{array}$ & Whiteflies & 1995 & $\mathrm{~L}$ \\
\hline Eretmocerus mundus & Hymenoptera & $\begin{array}{l}\text { Europe, Africa North and } \\
\text { South, North and Latin } \\
\text { America, Asia }\end{array}$ & Whiteflies & 2001 & $\mathrm{~L}$ \\
\hline Eretmocerus warrae & Hymenoptera & Aus/NZ & Whiteflies & 2000 & $\mathrm{~L}$ \\
\hline Euseius finlandicus & Acari & Europe & Mites & 2000 & $\mathrm{~S}$ \\
\hline Euseius scutalis & Acari & Europe & Mites & 1990 & $\mathrm{~S}$ \\
\hline Exochomus laeviusculus & Coleoptera & Europe & Aphids, scales & 1988 & $\mathrm{~S}$ \\
\hline $\begin{array}{l}\text { Exochomus } \\
\text { quadripustulatus }\end{array}$ & Coleoptera & Europe & Aphids, scales & 2000 & $\mathrm{~S}$ \\
\hline $\begin{array}{l}\text { Feltiella acarisuga } \\
\qquad(=\text { Therodiplosis persicae })\end{array}$ & Diptera & $\begin{array}{l}\text { Europe, North and Latin } \\
\text { America }\end{array}$ & Mites & 1990 & M \\
\hline $\begin{array}{l}\text { Franklinothrips megalops } \\
\quad(=\text { myrmicaeformis })\end{array}$ & Thysanoptera & Europe & Thrips & 1992 & $\mathrm{~S}$ \\
\hline Franklinothrips vespiformis & Thysanoptera & Europe, Asia & Thrips & 1990 & $\mathrm{~S}$ \\
\hline $\begin{array}{l}\text { Galendromus } \\
\quad \text { (Typhlodromus) } \\
\text { occidentalis }\end{array}$ & Acari & North America, Aus & Mites & 1969 & $\mathrm{~L}$ \\
\hline
\end{tabular}


Table 1 continued

\begin{tabular}{|c|c|c|c|c|c|}
\hline Natural enemy & Classification & Region where used & Target(s) & $\begin{array}{l}\text { Year of } \\
\text { first use } \\
\text { (estimated) }\end{array}$ & $\begin{array}{l}\text { Market } \\
\text { value }\end{array}$ \\
\hline $\begin{array}{l}\text { Galeolaelaps (Hypoaspis) } \\
\text { aculeifer }\end{array}$ & Acari & $\begin{array}{c}\text { Europe, Africa North, North } \\
\text { America, Asia, Aus/NZ }\end{array}$ & $\begin{array}{l}\text { Dipterans, thrips, } \\
\text { mites }\end{array}$ & 1995 & $\mathrm{~L}$ \\
\hline Geocoris punctipes & Heteroptera & North America & $\begin{array}{l}\text { Lepidopterans, } \\
\text { whiteflies }\end{array}$ & 2000 & $\mathrm{~S}$ \\
\hline Goniozus legneri & Hymenoptera & North America & Lepidopterans & 1990 & $\mathrm{~S}$ \\
\hline Gyranusoidea litura & Hymenoptera & Europe & Pseudococcids & 1990 & $\mathrm{M}$ \\
\hline Harmonia axyridis & Coleoptera & $\begin{array}{l}\text { Europe, except France where } \\
\text { wingless } H \text {. axyridis are used }\end{array}$ & Aphids & 1995-2005 & $\mathbf{L}$ \\
\hline Harmonia axyridis & Coleoptera & North America, Asia & Aphids & 1990 & $\mathrm{~L}$ \\
\hline $\begin{array}{r}\text { Heterorhabditis } \\
\text { bacteriophora }\end{array}$ & Nematoda & $\begin{array}{l}\text { Europe, Africa North, North } \\
\text { America, Aus }\end{array}$ & Coleopterans & 1984 & $\mathrm{~L}$ \\
\hline Heterorabditis bateriopora & Nematoda & Asia & Coleopterans & 2000 & $\mathrm{~L}$ \\
\hline Heterorhabditis megidis & Nematoda & Europe, North America & Coleopterans & 1990 & $\mathrm{~L}$ \\
\hline Heterorhabditis zealandica & Nematoda & Aus & Coleopterans & 1990 & $\mathrm{~L}$ \\
\hline Hippodamia convergens & Coleoptera & Europe & Aphids & 1993 & $\mathrm{~S}$ \\
\hline Hippodamia variegata & Coleoptera & Aus & Aphids & 2000 & $\mathrm{~S}$ \\
\hline Holobus flavicornis & Coleoptera & Europe & Mites & 2000 & $\mathrm{~S}$ \\
\hline $\begin{array}{l}\text { Iphiseius (Amblyseius) } \\
\text { degenerans }\end{array}$ & Acari & Europe, North America & Thrips & 1993 & $\mathrm{M}$ \\
\hline Kampimodromus aberrans & Acari & Europe & Mites & 1960-1990 & $\mathbf{S}$ \\
\hline Karnyothrips melaleucus & Thysanoptera & Europe & Diaspidids & 1985 & $\mathrm{~S}$ \\
\hline Lamyctinus coeculus & Chilopoda & Europe & Symphylans & 1995 & $\mathrm{~S}$ \\
\hline Leptomastidea abnormis & Hymenoptera & Europe, North America & Pseudococcids & 1984 & $\mathrm{~S}$ \\
\hline Leptomastix dactylopii & Hymenoptera & $\begin{array}{l}\text { Europe, Africa North, North } \\
\text { America }\end{array}$ & Pseudococcids & 1984 & M \\
\hline Leptomastix epona & Hymenoptera & Europe & Pseudococcids & 1992 & $\mathrm{~S}$ \\
\hline Leptomastix histrio & Hymenoptera & Europe & Pseudococcids & 1995 & $\mathrm{~S}$ \\
\hline Lixophaga diatraea & Diptera & Latin America & Lepidipterans & 1980 & $\mathrm{~L}$ \\
\hline Lydella minense & Diptera & Latin America & Coleopterans & 1990 & $\mathrm{~L}$ \\
\hline Lysiphlebus fabarum & Diptera & Europe & Aphids & 1990 & $\mathrm{~S}$ \\
\hline Lysiphlebus testaceipes & Hymenoptera & Europe & Aphids & 1990 & $\mathrm{~S}$ \\
\hline Macrocheles robustulus & Acari & Europe & $\begin{array}{l}\text { Dipterans, thrips, } \\
\text { lepidoptera, }\end{array}$ & 2010 & $\mathrm{~L}$ \\
\hline Macrolophus caligonisus & Heteroptera & Europe & $\begin{array}{l}\text { Whiteflies, } \\
\text { lepidopterans }\end{array}$ & 2005 & M \\
\hline $\begin{array}{l}\text { Macrolophus pygmaeus } \\
\text { (nubilis) }\end{array}$ & Heteroptera & $\begin{array}{l}\text { Europe, Africa North and } \\
\text { South }\end{array}$ & Whiteflies & 1994 & $\mathrm{~L}$ \\
\hline Mallada signata & Neuroptera & Aus & $\begin{array}{l}\text { Aphids, thrips, } \\
\text { lepidopterans, } \\
\text { mealybugs, } \\
\text { whiteflies, etc. }\end{array}$ & 2000 & $\mathrm{~L}$ \\
\hline Mesoseiulus longipes & Acari & North America & Mites & 1989 & $\mathrm{~L}$ \\
\hline Metaphycus flavus & Hymenoptera & Europe, North America & Coccids & 1995 & $\mathrm{~S}$ \\
\hline Metaphycus helvolus & Hymenoptera & Europe, Aus & Coccids & 1943 & $\mathrm{~S}$ \\
\hline $\begin{array}{l}\text { Metaphycus lounsburyi } \\
\quad \text { (bartletti) }\end{array}$ & Hymenoptera & Europe, Aus & Coccids & 1902 & $\mathrm{~S}$ \\
\hline
\end{tabular}


Table 1 continued

\begin{tabular}{|c|c|c|c|c|c|}
\hline Natural enemy & Classification & Region where used & Target(s) & $\begin{array}{l}\text { Year of } \\
\text { first use } \\
\text { (estimated) }\end{array}$ & $\begin{array}{l}\text { Market } \\
\text { value }\end{array}$ \\
\hline Metaphycus stanleyi & Hymenoptera & Europe & Coccids & 1990 & $\mathrm{~S}$ \\
\hline Metaphycus swirskii & Hymenoptera & Europe & Coccids & 1995 & $\mathrm{~S}$ \\
\hline Metaphycus spp. Peru & Hymenoptera & Latin America & Coccids & 1990 & $\mathrm{~S}$ \\
\hline Metaseiulus occidentalis & Acari & Europe & Mites & 1985 & $\mathrm{~S}$ \\
\hline Meteorus gyrator & Hymenoptera & Europe & Lepdipterans & 2005 & $\mathrm{~S}$ \\
\hline Micromus angulatus & Neuroptera & Asia & Aphids & 2005 & $\mathrm{~S}$ \\
\hline Micromus tasmaniae & Neuroptera & Aus/NZ & $\begin{array}{l}\text { Aphids, thrips, } \\
\text { lepidopterans, } \\
\text { whiteflies, etc. }\end{array}$ & 2000 & $\mathrm{~S}$ \\
\hline Microterys flavus & Hymenoptera & Europe & Coccids & 1987 & $\mathrm{~S}$ \\
\hline Microterys nietneri & Hymenoptera & Europe & Coccids & 1987 & $\mathrm{~S}$ \\
\hline Muscidifurax raptor & Hymenoptera & North and Latin America & Dipterans & 1970 & $\mathrm{~L}$ \\
\hline Muscidifurax raptorellus & Hymenoptera & Africa North, North America & Dipterans & 1970 & M \\
\hline Muscidifurax zaraptor & Hymenoptera & Europe, North America & Dipterans & 1982 & M \\
\hline Nabis pseudoferus ibericus & Heteroptera & Europe & Lepidopterans & 2009 & $S$ \\
\hline Nasonia vitripennis & Hymenoptera & Europe, North America & Dipterans & 1970 & $\mathrm{~S}$ \\
\hline Neochrysocharis formosa & Hymenoptera & Asia & Dipterans & 1990 & M \\
\hline $\begin{array}{l}\text { Neoseiulus (Amblyseius) } \\
\text { barkeri }\end{array}$ & Acari & Europe & Thrips & 1981 & $S$ \\
\hline $\begin{array}{l}\text { Neoseiulus (Amblyseius) } \\
\text { californicus }\end{array}$ & Acari & $\begin{array}{l}\text { Europe, Africa North and } \\
\text { South, North and Latin } \\
\text { America, Asia }\end{array}$ & Mites & 1985 & $\mathrm{~L}$ \\
\hline $\begin{array}{l}\text { Neoseiulus (Amblyseius) } \\
\text { cucumeris }\end{array}$ & Acari & $\begin{array}{c}\text { Europe, Africa North and } \\
\text { South, North and Latin } \\
\text { America, Asia, Aus/NZ }\end{array}$ & Thrips, mites & 1985 & $\mathrm{~L}$ \\
\hline $\begin{array}{l}\text { Neoseiulus (Amblyseius) } \\
\text { fallacis }\end{array}$ & Acari & Europe, North America & Mites & 1997 & $\mathrm{~S}$ \\
\hline Neoseiulus wearnei & Acari & Aus & Mites & 2000 & $\mathrm{~S}$ \\
\hline Nephus includens & Coleoptera & Europe & Pseudococcids & 2000 & $\mathrm{~S}$ \\
\hline Nephus reunioni & Coleoptera & Europe & Pseudococcids & 1990 & $\mathrm{~S}$ \\
\hline Nesidiocoris tenuis & Heteroptera & Europe, Africa North, Asia & $\begin{array}{l}\text { Whiteflies, } \\
\text { lepidopterans }\end{array}$ & 2003 & $\mathrm{~L}$ \\
\hline Ooencyrtus kuvanae & Hymenoptera & Europe & Lepidopterans & 1923 & $\mathrm{~S}$ \\
\hline Ooencyrtus pityocampae & Hymenoptera & Europe & Lepidopterans & 1997 & $\mathrm{~S}$ \\
\hline Ophelosia crawfordi & Hymenoptera & Europe & $\begin{array}{l}\text { Coccids, } \\
\text { pseudococcids, } \\
\text { margarodids }\end{array}$ & 1980 & $\mathrm{~S}$ \\
\hline Ophyra aenescens & Diptera & Europe, North America & Dipterans & 1995 & $\mathrm{~S}$ \\
\hline Opius pallipes & Hymenoptera & Europe & Dipterans & 1980 & $\mathrm{~S}$ \\
\hline Orgilus obscurator & Hymenoptera & Latin America & Lepidopterans & 1990 & $\mathrm{~L}$ \\
\hline Orius albidipennis & Heteroptera & Europe & Thrips & 1993 & $S$ \\
\hline Orius armatus & Heteroptera & Aus & Thrips & 1990 & $\mathrm{~S}$ \\
\hline Orius insidiosus & Heteroptera & Europe & Thrips & 1991-2000 & $\mathbf{L}$ \\
\hline Orius insidiosus & Heteroptera & North and Latin America & Thrips & 1985 & $\mathrm{~L}$ \\
\hline
\end{tabular}


Table 1 continued

\begin{tabular}{|c|c|c|c|c|c|}
\hline Natural enemy & Classification & Region where used & Target(s) & $\begin{array}{l}\text { Year of } \\
\text { first use } \\
\text { (estimated) }\end{array}$ & $\begin{array}{l}\text { Market } \\
\text { value }\end{array}$ \\
\hline Orius laevigatus & Heteroptera & Europe, Africa North, Asia & Thrips & 1993 & $\mathrm{~L}$ \\
\hline Orius majusculus & Heteroptera & Europe & Thrips & 1993 & M \\
\hline Orius minutus & Heteroptera & Europe & Thrips & 1993 & $\mathrm{~S}$ \\
\hline Orius strigicollis & Heteroptera & Asia & Thrips & 2000 & M \\
\hline Orius tristicolor & Heteroptera & Europe & Thrips & 1995-2000 & $\mathbf{S}$ \\
\hline Pachycrepoideus vindemiae & Hymenoptera & Latin America & Dipterans & 1980 & $\mathrm{~L}$ \\
\hline Paratheresia claripalpis & Hymenoptera & Latin America & Lepidopterans & 1980 & $\mathrm{~L}$ \\
\hline Pediobius foveolatus & Hymenoptera & North America & Coleopterans & 1980 & $\mathrm{~S}$ \\
\hline Peristenus digoneutis & Hymenoptera & North America & Heteropterans & 1980 & $\mathrm{~S}$ \\
\hline Pergamasus quisquiliarum & Acari & Europe & Symphylans & 2000 & $\mathrm{~S}$ \\
\hline $\begin{array}{r}\text { Phasmarhabditis } \\
\text { hermaphrodita }\end{array}$ & Nematoda & Europe & Snails & 1994 & $\mathrm{~S}$ \\
\hline Pheidole megacephala & Hymenoptera & Latin America & Colepterans & 1990 & $\mathrm{~L}$ \\
\hline Phytoseius finitimus & Acari & Europe & Mites & 2000 & $\mathrm{~S}$ \\
\hline Phytoseiulus longipes & Acari & Europe & Mites & 1990 & $\mathrm{~S}$ \\
\hline Phytoseiulus macropilis & Acari & Latin America & Mites & 1980 & $\mathrm{~L}$ \\
\hline Phytoseiulus persimilis & Acari & $\begin{array}{c}\text { Europe, Africa North and } \\
\text { South, North and Latin } \\
\text { America, Asia, Aus/NZ }\end{array}$ & Mites & 1968 & $\mathrm{~L}$ \\
\hline Picromerus bidens & Heteroptera & Europe & Lepidopterans & 1990 & $\mathrm{~S}$ \\
\hline Podisus maculiventris & Heteroptera & Europe, North America & $\begin{array}{l}\text { Coleopterans, } \\
\text { lepidopterans }\end{array}$ & 1996 & $\mathrm{~S}$ \\
\hline Podisus nigrispinus & Heteroptera & Latin America & Lepidopterans & 1990 & $\mathrm{~S}$ \\
\hline Praon volucre & Hymenoptera & Europe & Aphids & 1990 & $\mathrm{~S}$ \\
\hline Prorops nasuta & Hymenoptera & Latin America & Coleopterans & 1990 & $\mathrm{~L}$ \\
\hline Prospaltella spp. & Hymenoptera & Latin America & Diaspidids & 1990 & M \\
\hline Pseudaphycus angelicus & Hymenoptera & Europe & Pseudococcids & 1990 & $\mathrm{~S}$ \\
\hline Pseudaphycus flavidulus & Hymenoptera & Europe & Pseudococcids & 1990 & $\mathrm{~S}$ \\
\hline $\begin{array}{l}\text { Pseudaphycus } \\
\text { maculipennis }\end{array}$ & Hymenoptera & Europe & Pseudococcids & 1980 & $\mathrm{~S}$ \\
\hline Psyttalia concolor & Hymenoptera & Europe & Dipteran & 1968-2000 & $\mathbf{S}$ \\
\hline Rhyzobius chrysomeloides & Coleoptera & Europe & Coccids & 1980 & $\mathrm{~S}$ \\
\hline Rhyzobius forestieri & Coleoptera & Europe & Coccids & 1980 & $\mathrm{~S}$ \\
\hline $\begin{array}{l}\text { Rhyzobius (Lindorus) } \\
\text { lophanthae }\end{array}$ & Coleoptera & Europe, North America & Coccids & 1980 & $\mathrm{~S}$ \\
\hline Rodolia cardinalis & Coleoptera & Europe & Margarodids & 1990 & $\mathrm{~S}$ \\
\hline Rumina decollata & Mollusca & Europe, North America & Molluscs & 1990 & $\mathrm{~S}$ \\
\hline Saniosulus nudus & Acaridae & Europe & Diaspidids & 1990 & $\mathrm{~S}$ \\
\hline Scolothrips sexmaculatus & Thysanoptera & Europe, North America & Mites, thrips & 1990 & $\mathrm{~S}$ \\
\hline Scutellista caerulea (cyanea) & Hymenoptera & Europe & Coccids & 1990 & $\mathrm{~S}$ \\
\hline Scymnus rubromaculatus & Coleoptera & Europe & Aphids & 1990 & $\mathrm{~S}$ \\
\hline Spalangia cameroni & Hymenoptera & $\begin{array}{l}\text { Africa North, North and Latin } \\
\text { America }\end{array}$ & Dipterans & 1970 & $\mathrm{~S}$ \\
\hline
\end{tabular}


Table 1 continued

\begin{tabular}{|c|c|c|c|c|c|}
\hline Natural enemy & Classification & Region where used & Target(s) & $\begin{array}{l}\text { Year of } \\
\text { first use } \\
\text { (estimated) }\end{array}$ & $\begin{array}{l}\text { Market } \\
\text { value }\end{array}$ \\
\hline Spalangia endius & Hymenoptera & North and Latin America, Aus & Dipterans & 1970 & $\mathrm{~L}$ \\
\hline Spalangia gemini & Hymenoptera & North America & Dipterans & 1980 & $\mathrm{~S}$ \\
\hline Spalangia nigroaenea & Hymenoptera & North America & Dipterans & 1980 & $\mathrm{~S}$ \\
\hline Steinernema carpocapsae & Nematoda & $\begin{array}{l}\text { Europe, Africa North, North } \\
\text { America, Asia }\end{array}$ & Coleopterans & 1984 & M \\
\hline Steinernema glaseri & Nematoda & Europe & Coleopterans & 2002 & $\mathrm{~S}$ \\
\hline Steinernema feltiae & Nematoda & $\begin{array}{l}\text { Europe, Africa North and } \\
\text { South, North and Latin } \\
\text { America, Aus/NZ }\end{array}$ & Sciarids & 1984 & $\mathrm{~L}$ \\
\hline Steinernema kraussei & Nematoda & Europe, North America & Coleopterans & 2000 & $\mathrm{~S}$ \\
\hline Steinernema riobrave & Nematoda & North America & $\begin{array}{l}\text { Lepidopterans, } \\
\text { coleopterans }\end{array}$ & 2000 & $\mathrm{~S}$ \\
\hline Stethorus punctillum & Coleoptera & Europe, North America, Asia & Mites & 1984 & $\mathrm{~S}$ \\
\hline $\begin{array}{l}\text { Stratiolaelaps (Hypoaspis) } \\
\quad \text { miles }\end{array}$ & Acari & $\begin{array}{l}\text { Europa, North America, Aus/ } \\
\text { NZ }\end{array}$ & Sciarids & 1995 & $\mathrm{~L}$ \\
\hline $\begin{array}{l}\text { Stratiolaelaps (Hypoaspis) } \\
\text { scimitus }\end{array}$ & Acari & Europa, Latin America & Sciarids & 1990 & $\mathrm{~L}$ \\
\hline Sympherobius fallax & Neuroptera & Europe & Pseudococcids & 1994 & $\mathrm{~S}$ \\
\hline Synacra paupera & Hymenoptera & Europe & Sciarids & 2000 & $\mathrm{~S}$ \\
\hline Telenomus remus & Hymenoptera & Latin America & Lepidopterans & 1990 & $\mathrm{~L}$ \\
\hline $\begin{array}{l}\text { Tetracnemoidea } \\
\text { brevicornis } \\
(=\text { Hungariella pretiosa })\end{array}$ & Hymenoptera & Europe & $\begin{array}{l}\text { Pseudococcids, } \\
\text { margarodids }\end{array}$ & 1990 & $\mathrm{~S}$ \\
\hline $\begin{array}{l}\text { Tetracnemoidea peregrina } \\
\quad(=\text { Hungariella peregrina })\end{array}$ & Hymenoptera & Europe & $\begin{array}{l}\text { Pseudococcids, } \\
\text { margarodids }\end{array}$ & 1990 & $\mathrm{~S}$ \\
\hline $\begin{array}{l}\text { Tetrastichus coeruleus } \\
\quad \text { (asparagi) }\end{array}$ & Hymenoptera & Europe & Coleopterans & 2000 & $\mathrm{~S}$ \\
\hline Thripobius semiluteus & Hymenoptera & Europe, Aus & Thrips & 1995 & $\mathrm{~S}$ \\
\hline Trichogramma atopovirilia & Hymenoptera & Latin America & Lepidopterans & 1990 & $\mathrm{~S}$ \\
\hline Trichogramma bournieri & Hymenoptera & Africa South & Lepidopterans & 2001 & $\mathrm{~S}$ \\
\hline $\begin{array}{l}\text { Trichogramma brassicae } \\
\quad(=\text { maidis })\end{array}$ & Hymenoptera & Europe, North America & Lepidopterans & 1980 & $\mathrm{~S}$ \\
\hline Trichogramma cacoeciae & Hymenoptera & Europe & Lepidopterans & 1980 & $\mathrm{~S}$ \\
\hline Trichogramma carverae & Hymenoptera & Aus & Lepidopterans & 1990 & $\mathrm{~L}$ \\
\hline Trichogramma dendrolimi & Hymenoptera & Europe, Asia & Lepidopterans & 1950 & $\mathrm{~L}$ \\
\hline Trichogramma evanescens & Hymenoptera & Europe, Africa North, Asia & Lepidopterans & 1975 & $\mathrm{~L}$ \\
\hline Trichogramma exiguum & Hymenoptera & Latin America & Lepidopterans & 1990 & $\mathrm{~L}$ \\
\hline Trichogramma galloi & Hymenoptera & Latin America & Lepidopterans & 1980 & $\mathrm{~L}$ \\
\hline Trichogramma minutum & Hymenoptera & North America & Lepidopterans & 1970 & $\mathrm{~S}$ \\
\hline Trichogramma nerudai & Hymenoptera & Latin America & Lepidopterans & 1990 & $\mathrm{~S}$ \\
\hline Trichogramma ostriniae & Hymenoptera & North America, Asia & Lepidopterans & 1980 & $\mathrm{~S}$ \\
\hline Trichogramma pintoi & Hymenoptera & Latin America & Lepidopterans & 1990 & M \\
\hline Trichogramma platneri & Hymenoptera & North America & Lepidopterans & 1990 & $\mathrm{~S}$ \\
\hline Trichogramma pretiosum & Hymenoptera & North and Latin America, Aus & Lepidopterans & 1974 & $\mathrm{~L}$ \\
\hline
\end{tabular}


Table 1 continued

\begin{tabular}{|c|c|c|c|c|c|}
\hline Natural enemy & Classification & Region where used & Target(s) & $\begin{array}{l}\text { Year of } \\
\text { first use } \\
\text { (estimated) }\end{array}$ & $\begin{array}{l}\text { Market } \\
\text { value }\end{array}$ \\
\hline $\begin{array}{l}\text { Trichogrammatoidea } \\
\text { cryptophlebiae }\end{array}$ & Hymenoptera & Aus & Lepidopterans & 1990 & $\mathrm{~L}$ \\
\hline Typhlodromus athiasae & Acari & Europe & Mites & 1995 & $\mathrm{~S}$ \\
\hline Typhlodromus doreenae & Acari & Europe & Mites & 2003 & $\mathrm{~S}$ \\
\hline Typhlodromus pyri & Acari & Europe & Mites & 1990 & $\mathrm{~S}$ \\
\hline Typhlodromips montdorensis & Acari & Europe, Aus & Thrips, mites & 2003 & $\mathrm{~L}$ \\
\hline Urolepis rufipes & Hymenoptera & North America & Dipterans & 1990 & $\mathrm{~S}$ \\
\hline
\end{tabular}

Key: Market value: $\mathrm{L}=$ large (hundred thousand to millions of individuals sold per week), $\mathrm{M}=$ medium (ten thousand to a hundred thousand of individuals sold per week), $\mathrm{S}=$ small (hundreds to a few thousand of individuals sold per week); Africa North = North of Sahara, Africa South $=$ South of Sahara, North America $=$ Canada + USA, Aus = Australia, NZ = New Zealand; bold entries natural enemies: no longer in use

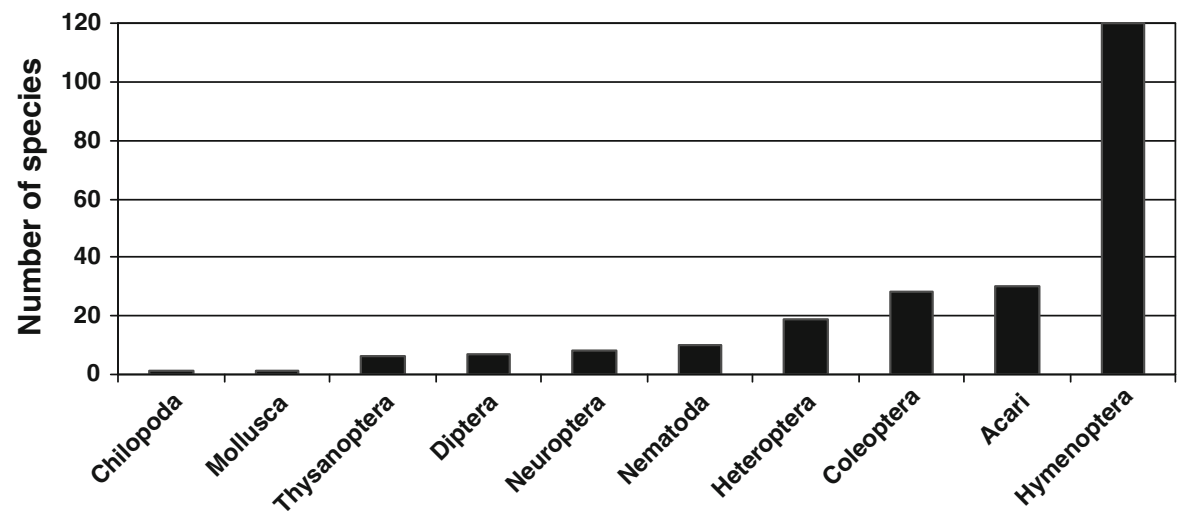

Fig. 1 Taxonomic groups providing natural enemies used in commercial augmentative biological control from 1900 to 2010

restricted host range, which is considered important in preventing undesirable side effects (e.g. Bigler et al. 2006). Acarid predators are popular because they can easily be mass reared, they can be released by mechanical means, may control several pest species, do not spread actively over large distances and are relatively small. The last two characteristics help to prevent negative effects on non-target species. An example of a recent acarid species becoming very popular in use is Amblyseius swirskii (Calvo and Belda 2007; Calvo et al. 2011).

The first species used in augmentative biological control were a hymenopteran (Metaphycus lounsburyi (bartletti)) and a coleopteran (Chilocorus circumdatus), both in 1902 (Table 1). During the initial seven decades of augmentative biological control (1900-1969) 11 species (0.11 year ${ }^{-1}$ ) became available, mainly hymenopterans (seven species). From 1970 onwards the number of new species becoming commercially available increased from 1.2 year $^{-1}$ (1970-1979), to 5.5 year $^{-1}$ (1980-1989), culminating in 10.9 year $^{-1}$ during the 1990 s, and decreased to 4.2 year $^{-1}$ during the first decade of the 21 st century (Fig. 2).

The strong increase in newly available natural enemies during the period 1970-1999 is caused by several factors. First of all, many pests developed resistance to insecticides after the initiation of large scale pesticide applications in the 1950s. This called for renewed use of already known biological control agents. And, if one natural enemy was used against an insecticide resistant pest, other pests in the same 


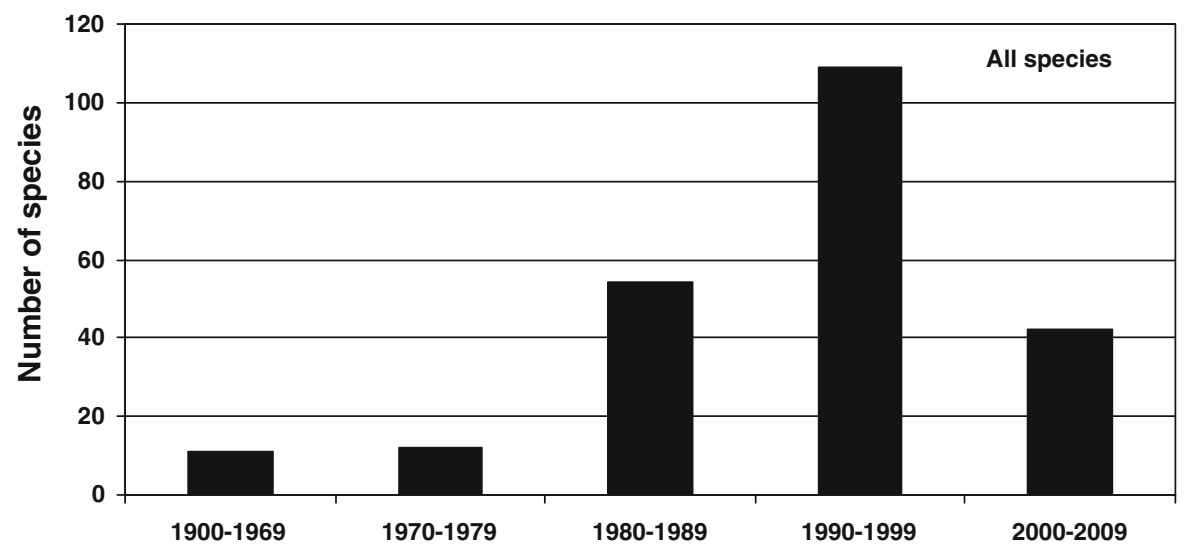

Fig. 2 Number of natural enemy species becoming available per time period

crop also needed to be managed with non-chemical control methods, including biological control (Hussey and Bravenboer 1971). This stimulated a search for new natural enemies and the development of Integrated Pest Management (IPM) (van Lenteren and Woets 1988; Parrella et al. 1999). For crops produced in greenhouses, biological control made it possible to use honey bees and bumble bees for pollination. Due to the great success of this type of pollination (reduced labour costs and, above all, increased production), growers were even more motivated to use biological control-not only for pests, but also for diseases (Albajes et al. 1999). At the same time, environmental and health concerns about pesticides encouraged design and implementation of IPM and biological control worldwide.

There are two reasons for the decrease in use of new natural enemy species after 2000: (1) efficient natural enemies were available for most of the pests in the agroecosystems where augmentative control is popular, and (2) stronger regulation of import of exotic natural enemies and registration of biological control agents has negatively affected their market penetration (Bolckmans 1999).

The relationship of number of species becoming available over time for the four taxonomic groups that provided most species of natural enemies is similar to that of all species combined (Fig. 3). They all show a peak during the period 1990-1999, with one obvious difference: heteropterans have only been used in augmentative control from 1990 onwards.

Most natural enemy species $(75 \% ; 173$ of 230 species) are produced in low or medium numbers per week (hundreds to a hundred thousand). This can be explained by their application in situations where only low numbers are needed (e.g. use in private gardens, hospitals, banks, shopping malls etc.), or the fact that they are only occasionally needed in large cropping systems (e.g. in greenhouses for control of minor pests). An example of a taxonomic group mainly used in niche markets is the Coleoptera, 26 of the 28 species are produced in small numbers (Table 1). Natural enemies produced in numbers of more than 100,000 per week ( $25 \%$; 57 of 230 species) can be separated in two groups: species where very large numbers need to be released per unit area in order to obtain sufficient control (acarids and nematodes), and species that are released at a low density on very large areas (coleopterans, heteropterans and hymenopterans in crops like citrus, sugar cane and maize). This means that simply looking at the numbers produced does not give a good indication of the market value of a species.

In Table 2 the 25 most often used natural enemy species are listed. These species make up more than $90 \%$ of the approximately $€ 300$ million of the total world market at end-user level (Bolckmans 2008; Cock et al. 2010). When expressed in sales volume, the most important commercial markets for natural enemies are greenhouse crops in The Netherlands, the UK, France and Spain, followed by the USA (Fig. 4). Together, these countries account for about twothirds of the total market (Bolckmans 1999). Nevertheless, Africa, Asia and Latin America represent significant and growing markets. The commercial market for field crops is tiny compared to the greenhouse market. 

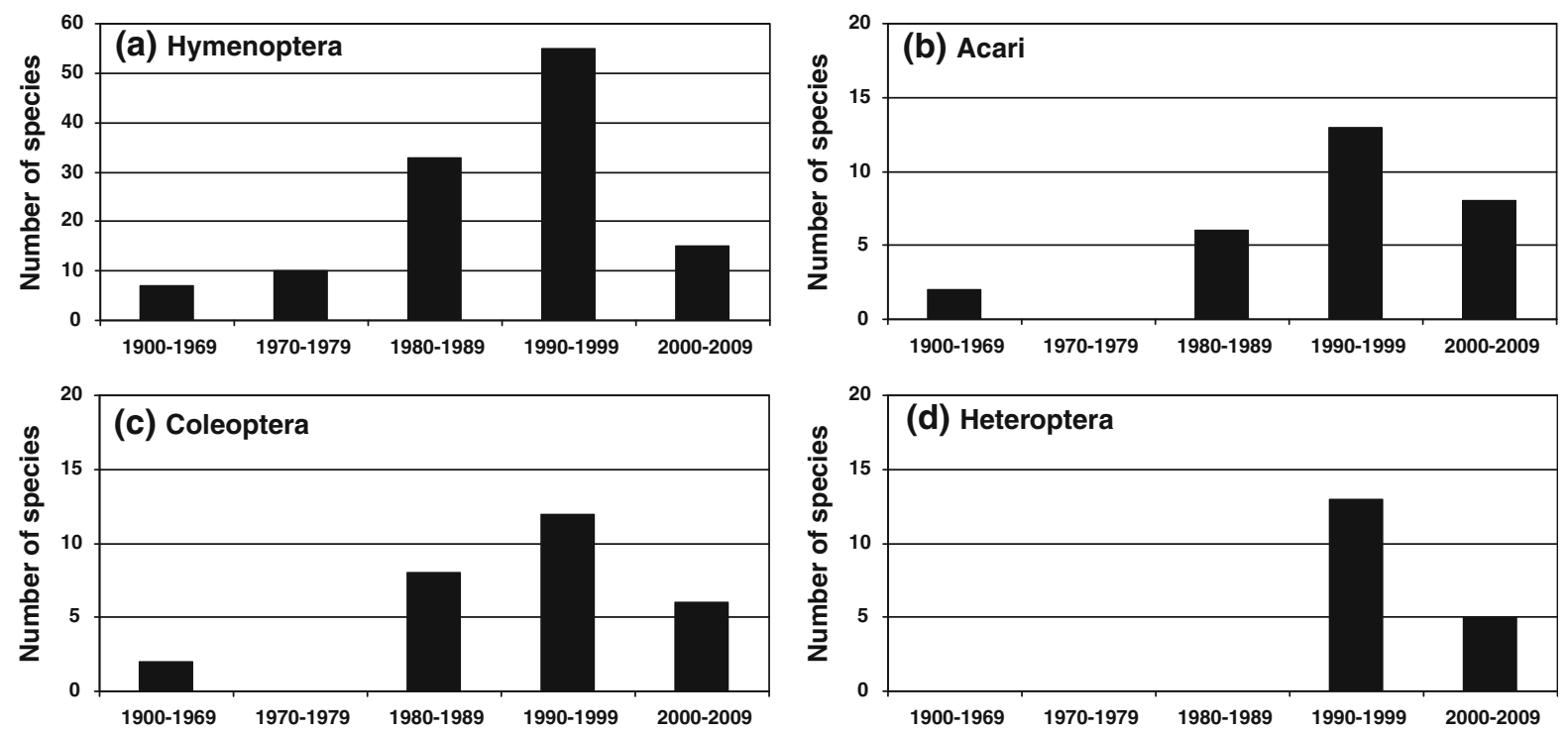

Fig. 3 Number of natural enemy species becoming available per time period for the four taxonomic groups which provided the majority of biological control agents: a Hymenoptera, b Acari, c Coleoptera and d Heteroptera

The agents used in augmentative biological control may be indigenous or exotic. Where they are exotic, they should-under best practice-undergo an environmental risk assessment, which is now common practice in several countries (Cock et al. 2010; van Lenteren et al. 2003, 2006). Due to the concern about import and release of exotic natural enemies and the increased evaluation and registration demands, there is a trend nowadays to first look for indigenous natural enemies when a new exotic pest establishes itself. This is clearly illustrated by the number of natural enemies that were used for the first time in Europe in previous decades (Fig. 5). Until 1970, the only two species commercially used in Europe were exotics. During the following three decades, more new exotic species (77) were used than indigenous species (58). In the last decade, this trend changed and for the first time more indigenous species (18) were commercialized than exotic species (6).

Of the natural enemy species commercially allowed for use in Africa, more than $90 \%$ results from material collected in and-initially mass reared on-other continents (Table 1). A similar situation exists in Canada, Japan, Mexico and South Korea. In Australia, New Zealand and the United States almost equal numbers of indigenous and exotic natural enemies are used. The situation is quite different in several South and Central American countries (e.g.
Argentina, Brazil and Cuba), where most of the natural enemies used in augmentative biological control are indigenous species (Table 1).

Table 1 shows another interesting development: not only are indigenous natural enemy species increasingly evaluated for first use, but also several of the popular exotic biological control agents have recently been replaced by indigenous species. The developments on the European market clearly illustrate this: nine exotic species have been substituted by indigenous species. Two important examples are the replacement of Eretmocerus eremicus by E. mundus and the replacement of Orius insidiosus by O. laevigatus.

\section{Commercial augmentative biological control: the current state of play}

Biological control is the most environmentally safe and economically profitable pest management method, which is illustrated by the data for biological and chemical control given in Table 3 . In biological control, we still have hundreds of thousands of species of natural enemies waiting to be discovered, and finding a new biological control agent is characterized by a very high success ratio compared to the ratio obtained in chemical control. In chemical control, 
Table 2 The most important invertebrate biological control agents used in augmentative biological control ranked by number of countries in which each is used (modified after Cock et al. 2010)

\begin{tabular}{|c|c|c|c|c|}
\hline Biological control agent & Family & Target(s) & $\begin{array}{l}\text { No. of countries } \\
\text { where used }\end{array}$ & $\begin{array}{l}\text { Year of } \\
\text { first use }\end{array}$ \\
\hline 1. Amblyseius swirskii & Phytoseiidae & Whiteflies, thrips, mites & $>20$ & 2005 \\
\hline 2. Aphidius colemani & Braconidae & Aphids & $>20$ & 1991 \\
\hline 3. Aphidoletes aphidimyza & Cecidomyiidae & Aphids & $>20$ & 1989 \\
\hline 4. Dacnusa sibirica & Braconidae & Leafminers & $>20$ & 1981 \\
\hline 5. Diglyphus isaea & Eulophidae & Leafminers & $>20$ & 1984 \\
\hline 6. Encarsia formosa & Aphelinidae & Whiteflies & $>20$ & 1926 \\
\hline 7. Macrolophus pygmaeus (=nubilis) & Miridae & Whiteflies & $>20$ & 1994 \\
\hline 8. Neoseiulus cucumeris (=Amblyseius cucumeris) & Phytoseiidae & Thrips & $>20$ & 1985 \\
\hline 9. Phytoseiulus persimilis & Phytoseiidae & Mites & $>20$ & 1968 \\
\hline 10. Steinernema feltiae & Steinernematidae & Sciarids & $>15$ & 1984 \\
\hline 11. Aphidius ervi & Braconidae & Aphids & $>15$ & 1996 \\
\hline 12. Orius laevigatus & Anthocoridae & Thrips & $>15$ & 1993 \\
\hline 13. Cryptolaemus montrouzieri & Coccinellidae & Coccids, pseudococcids & $>15$ & 1989 \\
\hline 14. Galeolaelaps aculeifer (=Hypoaspis aculifer) & Laelapidae & Sciarids & $>15$ & 1996 \\
\hline 15. Feltiella acarisuga (=Therodiplosis persicae) & Cecidomyiidae & Mites & $>15$ & 1990 \\
\hline 16. Leptomastix dactylopii & Encyrtidae & Pseudococcids & $>15$ & 1984 \\
\hline 17. Stratiolaelaps miles (=Hypoaspis miles) & Laelapidae & Sciarids & $>15$ & 1995 \\
\hline 18. Aphelinus abdominalis & Aphelinidae & Aphids & $>10$ & 1992 \\
\hline 19. Heterorhabditis bacteriophora & Heterorhabditidae & Coleopterans & $>10$ & 1984 \\
\hline 20. Heterorhabditis megidis & Heterorhabditidae & Coleopterans & $>10$ & 1990 \\
\hline 21. Neoseiulus californicus (=Amblyseius californicus) & Phytoseiidae & Mites, thrips & $>10$ & 1985 \\
\hline 22. Eretmocerus eremicus & Aphelinidae & Whiteflies & $>10$ & 1995 \\
\hline 23. Eretmocerus mundus & Aphelinidae & Whiteflies & $>10$ & 2001 \\
\hline 24. Episyrphus balteatus & Syrphidae & Aphids & $>10$ & 1990 \\
\hline 25. Trichogramma evanescens & Trichogrammatidae & Lepidopterans & $>10$ & 1975 \\
\hline
\end{tabular}

the success ratio has decreased from 1:50,000 in 1995 to 1:140,000 in 2008, while developmental costs have strongly increased during the past decades (McDougall 2010). The developmental costs for biological control are a fraction of those for chemical control. The time to develop a product is the same for both control methods. The benefit/cost ratio for inoculative biological control is much higher than for chemical control. For commercial augmentative biological control it is similar, but higher if we take indirect costs for chemical control into account which are related to environmental pollution and human health problems (Pimentel et al. 1980; Pimentel 2009). The risks of resistance are low or non-existent in biological control, while they are high in chemical control. High specificity and the lack of harmful side effects are characteristic for biological control agents.
More than 7,000 introductions involving almost 2,700 species of exotic arthropod agents for control of arthropod pests in 196 countries or islands during the past 120 years rarely have resulted in negative environmental effects (Cock et al. 2009, 2010), although there are some exceptions (Howarth 1991; Louda et al. 2003; van Lenteren et al. 2006) including the recent case of the Asian lady beetle (Harmonia axyridis) (van Lenteren et al. 2008). Application of chemical pesticides kills many species of nontarget organisms within and outside the agro-ecosystem, and may result in various side-effects, including unexpected, indirect and long-term effects on the environment and on the health of farmers and consumers (Pimentel et al. 1980; Pimentel 2009).

Before discussing the state of play in commercial augmentative biological control, I summarize the 


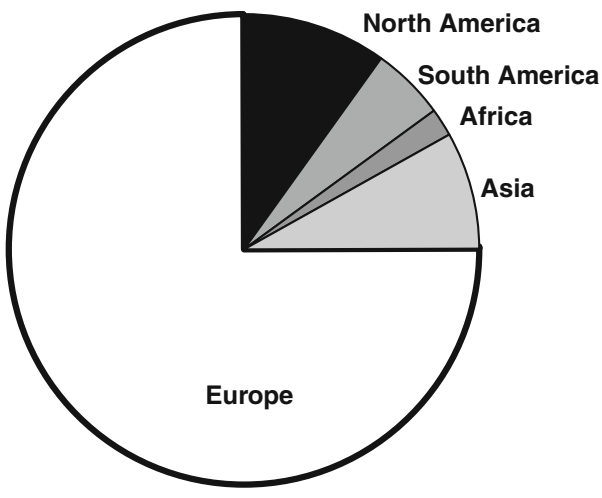

Fig. 4 The 2008 market share of commercial augmentative biological control by regions (after Cock et al. 2009)

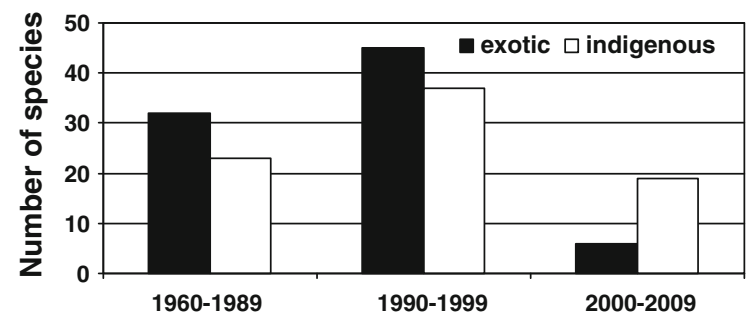

Fig. 5 Numbers of exotic (black) and indigenous (white) invertebrate natural enemies introduced to the European market over time

Table 3 Comparison of aspects related to the development and application of chemical and biological control

\begin{tabular}{lll}
\hline & $\begin{array}{l}\text { Chemical } \\
\text { control }^{\mathrm{a}}\end{array}$ & \begin{tabular}{l} 
Biological $_{\text {control }^{\mathrm{b}}}$ \\
\hline $\begin{array}{l}\text { Number of "ingredients" } \\
\text { tested }\end{array}$
\end{tabular} \\
$\begin{array}{l}\text { Success ratio } \\
\text { Developmental costs }\end{array}$ & $\begin{array}{l}\text { 256 million } \\
\text { Developmental time }\end{array}$ & 3,500 \\
$\begin{array}{l}\text { Benefit/cost ratio } \\
\text { Risks of resistance }\end{array}$ & $2: 1$ & $1: 10$ \\
Specificity & Large & 2 million US\$ \\
Harmful side-effects & Small & 10 years \\
\hline
\end{tabular}

${ }^{a}$ Main sources for data McDougall (2010), Pimentel et al. (1980), Pimentel (2009)

b Main sources for data Bale et al. (2008), Cock et al. (2009, 2010), Pimentel et al. (1980), Pimentel (2009)

situation for natural and inoculative control to make clear that biological control plays a very important role in today's agriculture and forestry. Natural biological control occurs on 89.5 billion ha of the world's ecosystems (land with vegetation), of which 44.4 billion ha is used for some form of agricultural activity (including forestry and grassland). Natural and inoculative biological control contribute to managing indigenous and alien pest problems in natural and managed ecosystems, and also in controlling vectors of human and veterinary diseases. The most widely used natural enemies in inoculative weed and insect control (e.g. Aphelinus mali, Aphytis lingnanensis, Cotesia flavipes, Cryptolaemus montrouzieri, Rodolia cardinalis, Teleonemia scrupulosa) have been introduced in more than 20 countries/regions worldwide and resulted in permanent control of the pest (Cock et al. 2009, 2010). Inoculative biological control is used on 350 million ha (10\% of land under cultivation). The "ecosystem service" provided by natural and inoculative biological control has an estimated value of at least 400 billion US\$ per year (Costanza et al. 1997), which is enormous, even when compared with the annual amount of 30 billion US\$ spent on chemical pest control (Crop Life International 2008). The impact of biological control is creating and sustaining public goods, such as food security, food quality, reduced pesticide use, human health (especially for farmers and farm workers), invasive alien species control, protection of biodiversity and maintenance of ecosystem services (Cock et al. 2010).

Compared to natural and inoculative biological control, commercial augmentative biological control is applied on a very limited scale, i.e. on only 16 million ha, which is $0.4 \%$ of cultivated land with crops on which this type of control could be used. Worldwide, some 30 "large" commercial producers are active (Bolckmans 2008), of which 20 are located in Europe. "Large" means that more than ten people are employed. In addition to these larger producers, it is estimated that about 500 small commercial producers are active. Fewer than five companies employ more than 50 people. The largest company has currently (2011) about 600 people employed. Producers organized themselves in different associations: in Europe in the International Biocontrol Manufacturers Association (IBMA), in North America in the Association of Natural Biocontrol Producers (ANBP), in Australia in Australasian Biological Control (ABC) and in Brazil in the Brazilian Association of Biological Control (ABCbio).

Given the large numbers of natural enemy species commercially available and the many positive 
characteristics of biological control specified above, the question emerges what the causes of the frustrating lack of uptake of augmentative biological control are. In the next section, the various and wide-ranging explanations for the limited use are discussed.

\section{Reasons for the limited use of commercial biological control}

Attitude of the pesticide industry

The pesticide industry is not interested in biological control, because natural enemies cannot be patented, cannot be stored for long periods, act very specifically, can often not be combined with chemical control and need extra training of sales personnel and farmers. The pesticide industry is not particularly concerned either with sustainable, long-term solutions for pest control as patent periods on pesticides are limited. Their concern is to develop and market new insecticides. This will be a continuous threat to biological control, although work of the International Organization for Biological Control (IOBC) has resulted in a European Union (EU) demand of testing side-effects on natural enemies for new pesticides. This knowledge tells which biological control agents will be killed when using certain pesticides. The sideeffect tests initially developed by IOBC and, later, in collaboration with the European Plant Protection Organization (EPPO) are now required elements of the EU registration procedure for pesticides (EPPO 2003). The attitude of the chemical industry has somewhat improved recently and some companies are even producing natural enemies. The reason for this is that the chemical industry desperately needs biological control, because with chemical control alone it is no longer possible to control all pests (Merino-Pachero 2007).

A more serious problem of pesticides is that they are unjustly cheap because society ends up paying for the so-called indirect costs created by pesticide use such as death of nontarget organisms, human health problems, environmental pollution and interference with ecosystem functions (Costanza et al. 1997; Pimentel 2009). Taking these costs into account, pesticides should be at least three times more expensive and, as a result, realistic pricing of pesticides would more often lead to a choice for biological control (Pimentel et al. 1980; Pimentel 2009).

Attitude of farmers

The current attitude of many farmers concerning pest control is that a crop cannot be grown without use of pesticides. This view is correct for many of the crops used in today's agriculture, because they have been selected under a blanket of pesticide applications with as main goal to identify cultivars with highest yields (food) or best appreciated cosmetic value (flowers). As a result, it will require a lot of creativity to accomplish a drastic change in the mind-set of pesticide addicted farmers and the replacement of poison dependent crop cultivars by disease and pest resistant crop cultivars.

Attitude of governmental institutions

Next, there is seldom a national or international policy to enforce the use of sustainable solutions for pest control. Farmers are of the opinion that registered pesticides are safe for the environment and for man, so there is no incentive for them to change. The industry, understandably, is not interested in complicated IPM systems with low profit margins. Therefore, it seems that only governments can effect change by enforcing use of non-chemical pest control (but see the remark below about the role of food retailers). European governments were provided as early as 1992 with important background information in the form of the report "Ground for Choices: four perspectives for the rural areas in the European Community" (Latesteijn 1992). This report showed that with good farming practices an overall reduction in pesticide volume used of more than $90 \%$ could be reached. In this same period, IOBC had developed and tested IPM programmes for a number of crops in which use of pesticides was even lower (e.g. van Lenteren et al. 1992). Although governments often react positively when asked how they think about biological and integrated control, such reactions can almost exclusively been put into the category "paying lip service", because financial, long-term support for research and implementation is not provided. One would expect governments to strongly stimulate use of environmentally friendly forms of pest management, because there is an overwhelming amount of 
information showing that agriculture is a major source of pollution and that chemical pesticides have serious negative effects on biodiversity and (natural) biological control. For example, Geiger et al. (2010) conclude: “... that despite decades of European policy to ban harmful pesticides, the negative effects of pesticides on wild plant and animal species persist, at the same time reducing the opportunities for biological pest control. If biodiversity is to be restored in Europe and opportunities are to be created for crop production utilizing biodiversity-based ecosystem services such as biological pest control, there must be a Europe-wide shift towards farming with minimal use of pesticides over large areas." Apparently, the tide is turning: the European Commission (EC) is aiming at replacement of chemical pesticides by non-chemical means of pest management (see below).

Influence of guidelines and regulations

Another factor frustrating application of biological control is the increasing amount of guidelines and regulations. Some of these regulations like the "Guidelines for the export, shipment, import and release of biological control agents and other beneficial organisms" (IPPC 2005), the guidelines for Environmental Risk Assessment mentioned earlier in this paper, and national regulations for import and release of biological control agents may delay implementation of biological control (Bolckmans 1999). Most of these guidelines could and should be drastically simplified and harmonized, which will result in application of more biological control. But the future of biological control might be really threatened by the plans concerning benefit sharing under the Convention of Biological Diversity (CBD). Under this convention countries have sovereign rights over their biodiversity. Agreements governing the access to these resources and the sharing of the benefits arising from their use need to be established between involved parties (i.e. Access and Benefit Sharing (ABS)). This also applies to species collected for potential use in biological control. Recent applications of CBD principles have already made it difficult or impossible to collect and export natural enemies for biological control research in several countries (Cock et al. 2010). The CBD was required to agree a comprehensive ABS process in 2010. In preparation for this, IOBC has prepared a position paper (Cock et al. 2010) in which the practice of biological control in relation to the principles of ABS is described and illustrated extensively by case studies and successes obtained with biological control. During the 10th Conference of the Parties to the Convention on Biological Diversity (18-29 October 2010, Nagoya, Japan) the Nagoya Protocol on Access to Genetic Resources and the Fair and Equitable Sharing of Benefits Arising from their Utilization to the Convention on Biological Diversity was adopted (CBD 2010; UN 2010). Article 8 'Special Considerations' of the Nagoya Protocol states (UN 2010):

In the development and implementation of its access and benefit-sharing legislation or regulatory requirements, each Party shall:

(a) Create conditions to promote and encourage research which contributes to the conservation and sustainable use of biological diversity, particularly in developing countries, including through simplified measures on access for non-commercial research purposes, taking into account the need to address a change of intent for such research;

(b) Pay due regard to cases of present or imminent emergencies that threaten or damage human, animal or plant health, as determined nationally or internationally. Parties may take into consideration the need for expeditious access to genetic resources and expeditious fair and equitable sharing of benefits arising out of the use of such genetic resources, including access to affordable treatments by those in need, especially in developing countries;

(c) Consider the importance of genetic resources for food and agriculture and their special role for food security.

Collection of natural enemies is not specifically mentioned as non-commercial research in this protocol and all countries involved in a natural enemy exploration project would have to prepare their legislation in such a way that exploration can be treated as non-commercial. It is expected that development of such legislation may form a time-consuming hurdle. If it is accepted that biological control is non-commercial research, simplified measures for ABS should facilitate biological control research. 
Furthermore, the use of biological control to address emergencies and the needs of food and agriculture should also be facilitated.

Attitude of biological control community

What about the biological control research community itself? Researchers and practitioners of biological control are not particularly good at lobbying and promoting the impressive benefits of biological control, they do not blow their own trumpet, they have not learned to defend their work forcefully, they often forget to illustrate the fantastic and permanent results obtained with inoculative biological control, thereby limiting discussions to the as yet restricted application of commercial biological control. Biological control workers are their own worst natural enemy!

It is also sad and disappointing that entomologists and biological control researchers have the peculiar habit of being over critical about their own work. An example is a publication by Collier and van Steenwyk (2004) with the title: "A critical evaluation of augmentative biological control". Yet, the article does not present an evaluation of augmentative biological control, but, instead, the authors evaluated some research articles, and neglected papers on practical application. What's more, the article is not a critical evaluation of augmentative biological control in general, but is mainly limited to a few experimental situations in the USA. There are, however, plenty examples of successful practical augmentative programs both within and outside the USA (see e.g. Gurr and Wratten 2000). Moreover, because the authors try to answer their questions with unsuitable data, their conclusions are in clear disagreement with the current state of affairs in the field of augmentative biological control illustrated above. An unforeseen aspect of such papers is that policymakers, politicians and the pesticide industry may use the-erroneous-information to show how poorly commercial biological control performs (van Lenteren 2006).

\section{Factors stimulating the use of biological control}

Next to the factors hampering application, there are significant developments which will stimulate the use of biological control.
Arthropod resistance to pesticides

Ongoing development of resistance to pesticides by arthropods, and increasing demands concerning the environmental and health effects of pesticides will make their development more difficult and costly. We can already see a stabilization and decrease of pesticide use in Europe and North America. As a result of the impossibility to control certain pests with chemicals, we see dramatic shifts from complete chemical control to mainly biological control in greenhouse vegetable production in North-West Europe, Spain and China, to name but a few (Merino-Pachero 2007; Pilkington et al. 2010).

Residue demands by food retailers and supermarket chains

An important development amongst food retailers and supermarket chains is that they are increasingly demanding pesticide poor or pesticide free food and prescribe pest management protocols to farmers. Supermarket chains, farmers and crop protection specialists collaborate in GLOBALGAP, a private sector body that sets standards for the certification of agricultural products around the globe. One of the GLOBALGAP guidelines concerns IPM, and biological control together with other types of non-chemical control form an essential part of this guideline (http://www.globalgap.org). These GLOBALGAP guidelines are often more restrictive about the use of conventional chemical pesticides than national or international regulations.

Attitude of consumers

Although it is generally believed that consumers prefer biological control above other pest control methods, this is rarely documented. In Canada, a professionally designed survey was conducted-a worldwide premiere-to determine the perception to the use of biological control as a means of pest management. The respondents clearly believed that foods produced using biological control were safer than those using synthetic insecticides. The majority of respondents felt that there would be less risk associated with consuming food when biological control agents, rather than synthetic chemical means, were used to control pests (McNeil et al. 2010). 
Change in attitude of governmental institutions

A recent example of how governmental institutions, in this case in Europe, can stimulate the use of biological control is the following. The European Commission (EC) is putting non-chemical forms of pest control high on the research and implementation agenda with the main goal to make agriculture less dependent on conservative synthetic chemical control (EU Directive 2009/128/EC; EC 2010). Again, due to EC policy, it is anticipated that 750 of 1,000 active ingredients used in chemical control will be phased out in the coming years. Furthermore, a substitution principle will be applied to new pesticides, by which the economically sound and environmentally safest agents will get priority for registration. In addition, each EC member country has to develop a national action program for IPM before 2014, and application of IPM, including biological control, will become the compulsory crop protection method from 2014 onwards. These measures are expected to form an important incentive for biological control.

This change in attitude currently perceived in Europe is, fortunately, not unique. In the past, environmental and health concerns have led to sustainable approaches of pest control in various areas worldwide as reviewed by Peshin et al. (2009).

Various other factors stimulating use of biological control

Implementation of biological control is assisted significantly by meeting the following basic conditions: (1) availability of a complete IPM programme for a crop, (2) total costs of IPM programme similar to costs for chemical control programme, (3) existence of reliable, independent extension service not compromised by the pesticide industry, and (4) support of application of non-chemical forms of pest management by governmental institutions. Another factor which helps implementation of biological control is the provision of sufficient and long term research funding. At present, funding of biological or IPM research is a minimal fraction $(<1 \%)$ of the funding for research in chemical control.

Application of the substitution principle whereby environmentally hazardous pesticides are replaced by the ecologically best alternatives will also increase application of biological control. In other cases, simply banning of the worst pesticides by governmental decree may lead to restoration of natural biological control (Kenmore 1991; Oka 1991) and provide better opportunities for augmentative biological control.

Moreover, realistic pricing of chemical control to compensate for indirect, societal and environmental costs (Pimentel 2009), would make the competition with sales of biological control agents fairer. In fact, several countries (e.g. Denmark, Norway, Sweden) are levying pesticide taxes, which are partly used for development of IPM programmes and incentives for farmers to encourage "low-pesticide" farming. These pesticide taxes also resulted in an immediate decrease in use of pesticides (Cannell 2007).

\section{Conclusions}

During the past 120 years, a large number of natural enemies has been collected and evaluated for use in augmentative biological control programmes. Particularly during the last 30 years many efficient species have been identified and currently at least 230 species are commercially available globally. Today, the commercial biological control industry is well organized, has developed mass production, shipment and release methods as well as adequate guidance for farmers. The industry has intensively collaborated with the public research sector in design of quality control programmes, which are applied during natural enemy production and shipment. The industry also cooperated in preparing environmental risk assessment methods for biological control agents. In several areas of agriculture augmentative biological control has obtained considerable successes and is now a reliable and appreciated element of IPM programmes. Despite all this progress, augmentative biological control is applied on a frustratingly small acreage.

Different reasons explain the slow uptake. The pesticide industry considers biological control as cumbersome and of restricted use, most farmers have become pesticide addicted during the past 60 years, governmental institutions do not enforce or stimulate non-chemical pest control, and many regulations concerning the collection and application of biological control agents delay or even prohibit their use.

Recent developments may, however, lead to a promising future for augmentative biological control. 
In addition to the ever ongoing development of resistance of pests to pesticides resulting in a need for alternative control methods, requirements of residue free food by supermarkets and consumers, prioritizing use of IPM by governmental institutions like the European Union and termination of pesticides subsidies, will all result in better possibilities for biological control. After 60 years of chemical control, we are entering the ecology-based pest management era!

Acknowledgments Part of this paper is influenced by discussions in and publications of the Commission on Biological Control and Access and Benefit Sharing of the International Organization for Biological Control (IOBCGlobal) (Cock et al. 2009, 2010). Many colleagues from all over the world are thanked for helping in collecting data about production and use of invertebrate natural enemies in augmentative biological control. Matthew Cock, Anke Bjelkeman-van Lenteren and two reviewers provided useful suggestions to improve the paper.

Open Access This article is distributed under the terms of the Creative Commons Attribution Noncommercial License which permits any noncommercial use, distribution, and reproduction in any medium, provided the original author(s) and source are credited.

\section{References}

Albajes R, Gullino ML, van Lenteren JC, Elad Y (eds) (1999) Integrated pest and disease management in greenhouse crops. Kluwer Publishers, Dordrecht

Bale J, van Lenteren JC, Bigler F (2008) Biological control and sustainable food production. Philos Trans R Soc Lond B 363(1492):761-776

Bigler F, Babendreier D, Kuhlmann U (2006) Environmental impact of invertebrates for biological control of arthropods: methods and risk assessment. CAB Int, Wallingford

Bolckmans KJF (1999) Commercial aspects of biological pest control in greenhouses. In: Albajes R, Gullino ML, van Lenteren JC, Elad Y (eds) Integrated pest, disease management in greenhouse crops. Kluwer Publishers, Dordrecht, pp 310-318

Bolckmans KJF (2008) De insectenfabriek. In: Osse J, Schoonhoven L, Dicke M, Buiter R (eds) Natuur als bondgenoot: biologische bestrijding van ziekten en plagen. Bio-Wetenschappen en Maatschappij, Den Haag, p 51

Calvo FJ, Belda JE (2007) Amblyseius swirskii, un depredador para el control de mosca blanca y trips en cultivos hortícolas. Phytoma España 190:58-62

Calvo FJ, Bolckmans K, Belda JE (2011) Control of Bemisia tabaci and Frankliniella occidentalis in cucumber by Amblyseius swirskii. BioControl 56:185-192

Cannell E (2007) European farmers plough ahead: pesticide use reduction. Pesticides News, 78, December 2007. http://www.pan-Europe.info. Accessed 16 March 2011
CBD (2010) Access to genetic resources and the fair and equitable sharing of benefits arising from their utilization. In: Convention on Biological Diversity, conference of the parties to the convention on biological diversity, 10th conference, Nagoya, Japan, 18-29 October 2010. http:// www.cbd.int/nagoya/outcomes. Accessed 16 March 2011

Cock MJW, van Lenteren JC, Brodeur J, Barratt BIP, Bigler F, Bolckmans K, Cônsoli FL, Haas F, Mason PG, Parra JRP (2009) The use and exchange of biological control agents for food and agriculture. Report prepared for the FAO Genetic Resources Commission by the IOBC Global Commission on Biological Control and Access and Benefit Sharing. IOBC, Bern

Cock MJW, van Lenteren JC, Brodeur J, Barratt BIP, Bigler F, Bolckmans K, Cônsoli FL, Haas F, Mason PG, Parra JRP (2010) Do new access and benefit sharing procedures under the convention on biological diversity threaten the future of biological control? BioControl 55:199-218. Supplementary material on online version (case studies, natural enemy releases, country views concerning ABS)

Collier T, van Steenwyk R (2004) A critical evaluation of augmentative biological control. Biol Control 31:245-256

Costanza R, d'Arge R, de Groot R, Farber S, Grasso M, Hannon B, Limburg K, Naeem S, O’Neill RV, Paruelo J, Raskin RG, Sutton P, van den Belt M (1997) The value of the world's ecosystem services and natural capital. Nature 387:253-260

Crop Life International (2008) Facts and figures - the status of global agriculture-2008. Crop Life International, Brussels

DeBach P (1964) Biological control of insect pests and weeds. Chapman and Hall, London

DeBach P, Rosen D (1991) Biological control by natural enemies, 2nd edn. Cambridge University Press, Cambridge

EC (2010) The CAP towards 2020: meeting the food, natural resources and territorial challenges of the future. http://ec.europa.eu/agriculture/cap-post-2013/communication/com2010-672_en.pdf. Accessed 16 March 2011

EPPO (2003) Environmental risk assessment scheme for plant protection products. Standard PP 3/9 of the European Plant Protection Organization. EPPO Bull 33:99-101

Geiger F, Bengtsson J, Berendse F, Weisser W, Emmerson M, Morales MB, Ceryngier P, Liira J, Tscharntke T, Winqvist C, Eggers S, Bommarco R, Part T, Bretagnolle V, Plantegenest M, Clement L, Dennis C, Palmer C, Onate JJ, Guerrero I, Hawro V, Aavik T, Thies C, Flohre A, Hanke S, Fischer C, Goedhart PW, Inchausti P (2010) Persistent negative effects of pesticides on biodiversity and biological control potential on European farmland. Basic Appl Ecol 11(2):97-105

Gurr GM, Wratten SD (2000) Measures of success in biological control. Kluwer Academic Publishers, Dordrecht

Howarth FG (1991) Environmental impacts of classical biological control. Annu Rev Entomol 36:485-509

Hussey NW, Bravenboer L (1971) Control of pests in glasshouse culture by the introduction of natural enemies. In: Huffaker CB (ed) Biological control. Plenum, New York, pp 195-216

IPPC (2005) Guidelines for the export, shipment, import and release of biological control agents and other beneficial organisms. International Standards for Phytosanitary 
Measures No. 3. Rome. Food and Agriculture Organization of the United Nations, International Standards for Phytosanitary Measures No. 3

Kenmore P (1991) How rice farmers clean up the environment, conserve biodiversity, raise more food, make higher profits. Indonesia's IPM - a model for Asia. FAO, Manila

Latesteijn H (ed) (1992) Ground for choices: four perspectives for the rural areas in the European Community. Netherlands Scientific Council for Government Policy. SDU uitgeverij, 's-Gravenhage

Louda SM, Pemberton RW, Johnson MT, Follett PA (2003) Nontarget effects: the Achilles' heel of biological control? Annu Rev Entomol 48:365-396

McDougall P (2010) The cost of new agrochemical product discovery: development and registration in 1995, 2000 and 2005-8; R\&D expenditure in 2007 and expectations for 2012. Consultancy study for Crop Life America and the European Crop Protection Association

McNeil JN, Cotnoir PA, Leroux T, Laprade R, Schwartz JL (2010) A Canadian national survey on the public perception of biological control. BioControl 55:445-454

Merino-Pachero M (2007) Almeria finally forced to turn green. Fruit Veg Technol 7:23-25

Oka IN (1991) Success and challenges of the Indonesian national integrated pest management programme in the rice based cropping system. Crop Prot 10:163-165

Parrella MP, Stengard Hansen L, van Lenteren JC (1999) Glasshouse environments. In: Bellows TS, Fisher TW (eds) Handbook of biological control. Academic Press, San Diego, pp 819-839

Peshin R, Bandral RS, Zhang WJ, Wilson L, Dhawan AK (2009) Integrated pest management: a global overview of history, programs and adoption. In: Peshin R, Dhawan AH (eds) Integrated pest management: innovation-development process. Springer, Dordrecht, pp 1-49

Pilkington LJ, Messelink G, van Lenteren JC, Le Mottee K (2010) Protected biological control, biological pest management in the greenhouse industry. Biol Control 52: 216-220

Pimentel D (2009) Environmental and economic costs of the application of pesticides primarily in the United States. In: Peshin R, Dhawan AK (eds) Integrated pest management: innovation-development process. Springer, Dordrecht, pp 89-111

Pimentel D, Andow D, Dyson-Hudson R, Gallahan D, Jacobson S, Irish M, Kroop S, Moss A, Schreiner I, Shephard M, Thompson T, Vinzant B (1980) Environmental and social costs of pesticides: a preliminary assessment. Oikos 34:126-140

UN (2010) Nagoya protocol on access to genetic resources and the fair and equitable sharing of benefits arising from their utilization to the convention on biological diversity. http://treaties.un.org/doc/source/signature/2010/CN782E. pdf. Accessed 30 June 2011 van Lenteren JC (ed) (2003) Quality control and production of biological control agents: theory and testing procedures. CABI Publishing, Wallingford, UK

van Lenteren JC (2006) How not to evaluate augmentative biological control. Biol Control 39:115-118

van Lenteren JC, Bueno VHP (2003) Augmentative biological control of arthropods in Latin America. BioControl 48:123-139

van Lenteren JC, Godfray HCJ (2005) European science in the Enlightenment and the discovery of the insect parasitoid life cycle in The Netherlands and Great Britain. Biol Control 32:12-24

van Lenteren JC, Woets J (1988) Biological and integrated pest control in greenhouses. Annu Rev Entomol 33:239-269

van Lenteren JC, Minks AK, de Ponti OMB (eds) (1992) Biological control and integrated crop protection: towards environmentally safer agriculture. Pudoc, Wageningen

van Lenteren JC, Babendreier D, Bigler F, Burgio G, Hokkanen HMT, Kuske S, Loomans AJM, Menzler-Hokkanen I, van Rijn PCJ, Thomas MB, Tomassini MC, Zeng QQ (2003) Environmental risk assessment of exotic natural enemies used in inundative biological control. BioControl 48:3-38

van Lenteren JC, Bale J, Bigler F, Hokkanen HMT, Loomans AJM (2006) Assessing risks of releasing exotic biological control agents of arthropod pests. Annu Rev Entomol 51:609-634

van Lenteren JC, Bigler F, Babendreier D, Loomans AJM (2008) Harmonia axyridis: an environmental risk assessment for Northwest Europe. BioControl 53:37-54

Waage JK, Greathead DJ (1988) Biological control: challenges and opportunities. Philos Trans R Soc Lond B 318: $111-128$

\section{Author Biography}

Joop C. van Lenteren works since 1970 on behavioural ecology and population dynamics of parasitoids, theoretical and practical aspects of biological control, IPM and sustainable crop production, and anatomy and sensory physiology of parasitoid ovipositors. He is Emeritus Professor of Entomology at Wageningen University in the Netherlands. He has been and is active in various functions in the International Organization for Biological Control since 1974. His current activities include design and testing of pre-release evaluation criteria for natural enemies, development of environmental risk assessment methods, and work on access and benefit sharing in biological control. 